\title{
Roman Nedela
}

Locally homogeneous graphs with dense links at vertices

Czechoslovak Mathematical Journal, Vol. 42 (1992), No. 3, 515-517

Persistent URL: http://dml.cz/dmlcz/128344

\section{Terms of use:}

(C) Institute of Mathematics AS CR, 1992

Institute of Mathematics of the Czech Academy of Sciences provides access to digitized documents strictly for personal use. Each copy of any part of this document must contain these Terms of use.

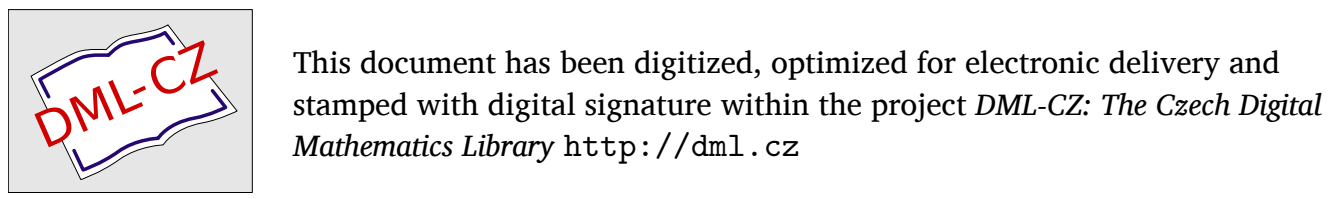




\title{
LOCALLY HOMOGENEOUS GRAPHS WITH DENSE LINKS
}

\section{AT VERTICES}

\author{
Roman Nedela, Banská Bystrica
}

(Received March 29, 1991)

Let $G$ be a locally finite graph and $u$ its vertex. Denote by link $(u, G)$ the subgraph of $G$ induced on the set of vertices adjacent to $u$. Let $G_{0}$ be a finite graph. The graph $G$ is called locally $G_{0}$, or locally homogeneous, if link $(u, G) \cong G_{0}$ for each vertex $u$ of $G$.

The original motivation for the study of locally homogeneous graphs probably came from the theory of finite automata. At one of the first conferences on graph theory, held in Smolenice in 1963, Zykov [9] formulated the following two problems:

(P1) Which are the finite graphs $G_{0}$ that there is a locally $G_{0}$ graph $G$ ?

(P1F) Which are the finite graphs $G_{0}$ that there is a finite locally $G_{0}$ graph $G$ ?

Another problem related to locally homogeneous graphs reads as follows:

(P2) For a given finite graph $G_{0}$ characterize all graphs $G$ that are locally $G_{0}$.

A survey paper [3] dealing with (P1) and (P1F) was published in 1976. New information on the research related to (P1), (P1F) and (P2) can be found in [4].

It is quite a simple observation that the complete graph $K_{n+1}$ is the only locally $K_{n}$ graph. The complete graph $K_{n}$ has the maximum number of edges among all $n$-vertex graphs. We say that a locally $G_{0}$ graph has a dense link if the number of edges of $G_{0}$ is great in comparison with the number of its vertices. The example of a complete graph suggests that the structure of the graph $G$ is strongly determined by its link provided $G_{0}$ is dense. In this note we shall present two ways how to make this sentence more precise. The first way comes from the following result of Soltés [7]. We shall call a graph $G$ an $(r, t)$-graph if there are integers $r>t \geqslant 0$ such that $G$ is $r$-regular and the link of each vertex is a $t$-regular graph. It is easy to see that $G$ is an $(r, t)$-graph if and only if $G$ is $r$-regular and each edge of $G$ lies in $t$ triangles. 
Nice examples of $(r, t)$-graphs are strongly regular graphs. It should be noted that an $(r, t)$-graph is not necessarily locally homogeneous. In [7] Soltés proved the following

Theorem 1 (Šltés). Let $t$ and $r$ be integers such that $t<r<t+\sqrt{\frac{8}{9}(t-1)}+\frac{4}{3}$ holds. Then a connected finite graph $G$ is an $(r, t)$-graph if and only if $r-t$ divides $r$ and $G$ is the complete $\left(1+\frac{r}{r-t}\right)$-partite graph whose each part has $r-t$ vertices.

As a consequence we obtain

Corollary 2. Let $G_{0}$ be a $t$-regular graph with $r$ vertices such that $t<r<$ $t+\sqrt{\frac{8}{9}(t-1)}+\frac{4}{3}$ holds. Then a connected finite graph $G$ is locally $G_{0}$ if and only if $r-t$ divides $r$ and $G$ is the complete $\left(1+\frac{r}{r-t}\right)$-partite graph whose each part has $r-t$ vertices.

Let $t \geqslant 1$ be a square. Then the tensor product $K_{2+\sqrt{t}} \otimes K_{2+\sqrt{t}} \cong G$ is locally $K_{1+\sqrt{t}} \otimes K_{1+\sqrt{t}}$ and $G$ is $t+2 \sqrt{t}+1$ regular. Thus the coefficient at $\sqrt{t-1}$ in Theorem 1 and Corollary 2 cannot exceed 2 .

The second way of making the notion of a dense link in a graph precise was motivated by the following result of Bugata, Horñak and JendroP [2]. If $H$ is a graph then $\bar{H}$ denotes its complement.

Theorem 3 (Bugata, Horn̆ák, Jendrol). Let $T$ be a tree. Then a locally $\bar{T}$ graph exists if and only if $T$ is a path or a star.

The main ideas of the proof of the following theorem were extracted from the proof of Theorem 3 in [2].

Theorem 4. Let $H$ be a graph satisfying the following two conditions:

(i) there are two adjacent vertices in $H$ whose degrees differ by at least 2,

(ii) each 4-vertex induced subgraph of $H$ contains at least two adjacent edges.

Then there is no locally $H$ graph.

Proof. Suppose there is a locally $H$ graph $G$. Let $v$ be a vertex in $G$ and let $x$, $y$ be vertices in link $(v, G)$ such that $\operatorname{deg}_{H} x+2 \leqslant \operatorname{deg}_{H} y$. Since $G$ is $v(H)$-regular there are two vertices $w, z$ in $V(G)-(\operatorname{link}(v, G) \cap\{v\})$ adjacent to $x$ but not to $y$. Any of the vertices $y$, and $v$ is adjacent neither to $w$ nor to $z$. Thus there are no two adjacent edges in the subgraph of $G$ induced on the vertices $v, y, w, z$. However, all these vertices belong to link $(x, G)$, contradicting the assumption. 
It is easy to see that conditions (i) and (ii) can be replaced by the following two conditions for the complementary graph:

(j) there are two non-adjacent vertices in $\bar{H}$ whose degrees differ by at least 2 ,

(jj) $\bar{H}$ contains no quadrangle.

Since each tree which is not a path or a star contains a pendant vertex $v$ and a vertex of a degree $\geqslant 3$ nonadjacent to $v$, one implication in Theorem 3 follows.

Theorem 4 is in a sense complementary to Corollary 2 because it covers irregular cases (but not all) of locally homogeneous graphs with a dense link.

Since the cartesian product $K_{p} \times K_{2}$ is locally the disjoint union $K_{p-1} \cup K_{1}$, by [8] we have for every finite group $\Gamma$ a locally $K_{p-1} \cup K_{1}$ graph $G$ such that $\Gamma \cong \operatorname{Aut}(G)$. This means that we must be careful in an attempt to formulate more precisely the above mentioned idea that the structure of a locally homogeneous graph with a dense link is more strongly determined by its link than in the case of a thin link.

In this note we were interested in locally homogeneous graphs with dense links at vertices. One can believe that if, on the other hand, the number of edges at a link $G_{0}$ of a vertex in a locally homogeneous graph $G$ is small, then the structure of $G$ is more free that it is if $G_{0}$ is dense. The results of the papers $[5,6]$ support this idea.

Concluding remark. It paid on my attention that Brouwer, Cohen and Neumaier in [1; Theorem 1.4.3] proved that an $(r, t)$-graph with $\sqrt{r}+\frac{1}{2} \geqslant r-t$ is complete multipartite. Since $\sqrt{r}+\frac{1}{2}>\sqrt{t}+\frac{1}{2}>\sqrt{\frac{8}{9}(t-1)}+\frac{4}{3}$, this result implies Theorem 1 .

\section{References}

[1] A. E. Brouwer, A. M. Cohen, A. Neumaier: Distance-regular graphs, Springer-Verlag, Berlin, 1989.

[2] P. Bugata, M. Horñák, S. Jendrol: On graphs with given neighborhoods, Casopis pro pèstování matematiky 2 (1989), 146-154.

[3] P. Hell: Graphs with given neighbourhoods I, Problémes combinatories at théorie des graphes, Proc, Coll. Int. C.N.R.S., Orsay, 1976, pp. 219-223.

[4] R. Nedela: Locally homogeneous graphs. Thesis, Faculty of mathematics and physics. Comenius University, Bratislava, 1991.

[5] R. Nedela: Morphisms of graphs preserving its local structure, Submitted.

[6] R. Nedela: Covering spaces of locally homogeneous graphs, Submitted.

[7] $L$ '. Soltés: Regular graphs with regular neighbourhoods, to appear.

[8] W. Vogler: Representing groups by graphs with constant link and hypergraphs, J. Graph Theory 10 (1986), 461-475.

[9] A. A. Zykov: Problem 30. in Theory of graphs and its applications, Proc Symp. Smolenice 1963, Praha, 1964, pp. 164-165.

Author's address: Pedagogická fakulta, Tajovského 40, 97549 Banská Bystrica. 